\title{
Enhanced Discrimination of Novel, Highly Similar Stimuli by Adults with Autism During a Perceptual Learning Task
}

\author{
Kate Plaisted, Michelle O'Riordan, and Simon Baron-Cohen \\ University of Cambridge, U.K.
}

\begin{abstract}
High-functioning adults with autism and control adults were tested on a perceptual learning task that compared discrimination performance on familiar and novel stimuli. Control adults were better able to discriminate familiar than novel stimuli-the perceptual learning effect. No perceptual learning effect was observed in adults with autism although they discriminated the novel stimuli significantly better than control adults. This enhanced discrimination learning about novel, but not familiar, stimuli in autism is discussed in relation to two current hypotheses of information processing in autism-weak central coherence and reduced attention-switching - and a new third hypothesis, which suggests that features held in common between stimuli are processed poorly and features unique to a stimulus are processed well in autism.
\end{abstract}

Keywords: Autism, attention, learning.

\section{Introduction}

It is frequently observed that individuals with autism show poor transfer of newly acquired skills or knowledge from a training context to a novel environment (Mirenda \& Donnellan, 1987; Woods, 1987), and parents of individuals with autism often comment that the behaviour of their children at school or in structured educational environments can be quite unlike their behaviour at home. Children with autism are also often attributed with obsessive attentional focus on specific stimuli in their environments. It has therefore been suggested that individuals with autism select only a small subset of the total stimulus array upon which to base newly acquired responding (Lovaas, Schreibman, Koegel, \& Rhem, 1971). As a result, transfer to new environments is limited because those incidental stimuli upon which responding is based may not be present in the transfer situation.

This overselectivity hypothesis has been extensively researched (see Lovaas, Koegel, \& Schreibman, 1979, for a review), and it has often been found that when certain stimuli that were present in the training environment are introduced into the transfer environment, generalisation of learning is substantially improved (e.g. Rincover \& Koegel, 1975). However, the problem with this as an analysis of poor transfer in autism is that a similar lack of generalisation following training frequently occurs in groups of individuals with moderate to severe learning disabilities $^{1}$ (Donnellan \& Mirenda, 1983), and these

Requests for reprints to: K. C. Plaisted, Department of Experimental Psychology, University of Cambridge, Downing Site, Cambridge CB2 3EB, U.K.

(E-mail: kcp1000@cus.cam.ac.uk). individuals also show stimulus overselectivity (Wilhelm $\&$ Lovaas, 1976). The overselectivity hypothesis therefore addresses the problem of poor transfer in children with autism as it relates to their associated learning disability rather than to their autism. Although this does not undermine the substantial importance of the hypothesis in isolating variables mediating poor transfer in populations with learning disabilities, it does not isolate those variables, mediating poor transfer, which are specific to autism.

Other training studies in which poor transfer of newly acquired knowledge does appear to be related to autism rather than learning disability are those in which children with autism are taught mental states terms. Ozonoff and Miller (1995), for example, gave a group of highfunctioning children with autism (whose full scale IQ was well within one standard deviation of the normal population mean) extensive group training on general social skills and the sorts of perspective-taking skills required in theory of mind tasks. Although the study showed that this training produced improvements in formal measures of theory of mind (e.g. M\&M's False Belief TaskPerner, Frith, Leslie, \& Leekam, 1989), indicating that children with autism can learn perspective-taking, the children showed no transfer of training to general social competence according to parents' and teachers' ratings.

Another study has explicitly compared transfer of learning by children with learning disabilities and children with autism. Swettenham (1996) trained children with autism, children with Down's syndrome, and typically developing children to solve a computerised version of the Sally Ann false belief task (Baron-Cohen,

1 The term "learning disability" is used here in accordance with the U.K. usage to refer to people of low general intelligence, in preference to the previous term "mental handicap". 
Leslie, \& Frith, 1985). When all three groups of children had successfully learned the task, they were then given two types of transfer test-close transfer tests (two versions of the Sally Ann task) and distant transfer tasks (three other false belief tasks involving other stimuli). Although there was no difference in performance between the three groups on the close transfer tasks, the children with autism failed all three distant transfer tasks, whereas the children with Down's syndrome performed as well as the typically developing children. This study is therefore a clear demonstration of lack of transfer due to autism rather than to learning disability.

One explanation of the poor transfer of training to more realistic social situations (Ozonoff \& Miller, 1995) or to distant transfer tasks, which involve different materials to the trained task (Swettenham, 1996), is that individuals with autism are unable to learn to reason about others' mental states. Thus, the children in Swettenham's study, for example, may have shown good transfer to the two new versions of the Sally Ann task because they had learnt a non-mentalising strategy specific to that task. But an equally plausible alternative explanation of the poor transfer is that the children with autism failed to respond to those few features held in common between the training and transfer situations. After all, the distant transfer tasks and real-life social situations involved many novel stimuli whose features were quite different to those present during training, and few familiar stimuli that held features in common with stimuli in the training situation. Transfer under these conditions will be successful if the subject processes those features that are held in common between the two situations rather than the novel, unique features.

This raises the possibility that these effects arise from a difference in the way in which people with and without autism process those features that are unique to a stimulus or a situation compared to how they process those features that are held in common between stimuli or situations. Specifically, our hypothesis is that individuals with autism process unique features extremely well and process common features poorly relative to nonautistic individuals. This predicts that individuals with autism will perform poorly on tasks that require the same response to two situations, where those situations share few common features and each contains many unique features, and that they should show the reverse by performing well on tasks that are complementary opposites - tasks that require a different response to two situations sharing many common features and few unique features. A discrimination task involving similar stimuli is one such task.

We therefore examined the hypothesis that there are differences in the way in which responding is controlled by unique and common features between individuals with and without autism using a difficult discrimination task - a perceptual learning task. Perceptual learning is the phenomenon whereby two similar stimuli, which upon first encounter appear identical, become discriminable following repeated presentation (E. J. Gibson, 1969; Hall, 1991; McLaren, Kaye, \& Mackintosh, 1989). An everyday example of perceptual learning is birdwatching - the novice birdwatcher is, at first, quite unable to tell apart two species of bird (such as a Coal Tit and a
Great Tit) that hold many visual characteristics in common, but becomes quite expert at discriminating between their slight differences after some exposure.

In the standard perceptual learning task, the subject is exposed to two highly similar stimuli (preexposure phase) and is subsequently required to learn to discriminate between them (test phase). In the test phase, the subject is also required to discriminate between two further novel stimuli, which are also highly similar. Perceptual learning is said to have occurred when the preexposed stimuli are better discriminated than the novel, non-preexposed stimuli. This effect has been observed in animals (e.g. Aitken, Bennett, McLaren, \& Mackintosh, 1996; E. J. Gibson \& Walk, 1956), in children (e.g. Attneave, 1957; J. J. Gibson \& Gibson, 1955), and in adults (e.g. McLaren, Leavers, \& Mackintosh, 1994).

In order to understand why two highly similar stimuli are difficult to discriminate, and why they become discriminable following preexposure, it is useful to represent each in terms of sets of features, as illustrated in Fig. 1. Each stimulus consists of two subsets of features: one small subset unique to itself ("a " features in the case of Stimulus A and " $b$ " features in the case of Stimulus B), and another, larger, subset that are present in, or common to, both stimuli (" $x$ " features). Associative learning theory assumes that generalisation between two such stimuli will depend on the relative proportion of common to unique elements - more common features and fewer unique features will result in greater generalisation. There are several demonstrations in animal learning experiments that this assumption is true: animals conditioned to one stimulus (such as a solution tasting of sucrose and lemon) will show more generalisation of that conditioning to another similar stimulus which shares a common feature (such as a solution tasting of saline and lemon) than to a less similar stimulus (such as a saline-alone solution-Mackintosh, Kaye, \& Bennett, 1991; Pearce \& Redhead, 1993).

Of course, the greater the generalisation between two stimuli, the harder they are to discriminate. If a subject was required to learn a discrimination between two similar stimuli- to learn to respond in one way to one stimulus and in another way to the other - this would be a difficult task, because the larger set of " $x$ " features, being present in both stimuli, would be associated equally with each of the two responses. In order to solve this discrimination, the subject would have to learn to associate the few unique features of each stimulus with the appropriate response.

However, the perceptual learning effect-better discrimination between preexposed stimuli during the test phase compared to non-preexposed stimuli-shows that processes must occur during the preexposure phase that reduce the salience of the shared " $\mathrm{x}$ " features, allowing the unique elements of each stimulus to be learned about more easily in the subsequent discrimination phase. Hence, these processes reduce generalisation of responding between the stimuli during discrimination learning in the test phase. That is, the subject is more able to associate the unique elements of one stimulus with one response and the unique elements of the other with an alternative response: they are treated as different stimuli. (See McLaren et al., 1989, for a full discussion of the 


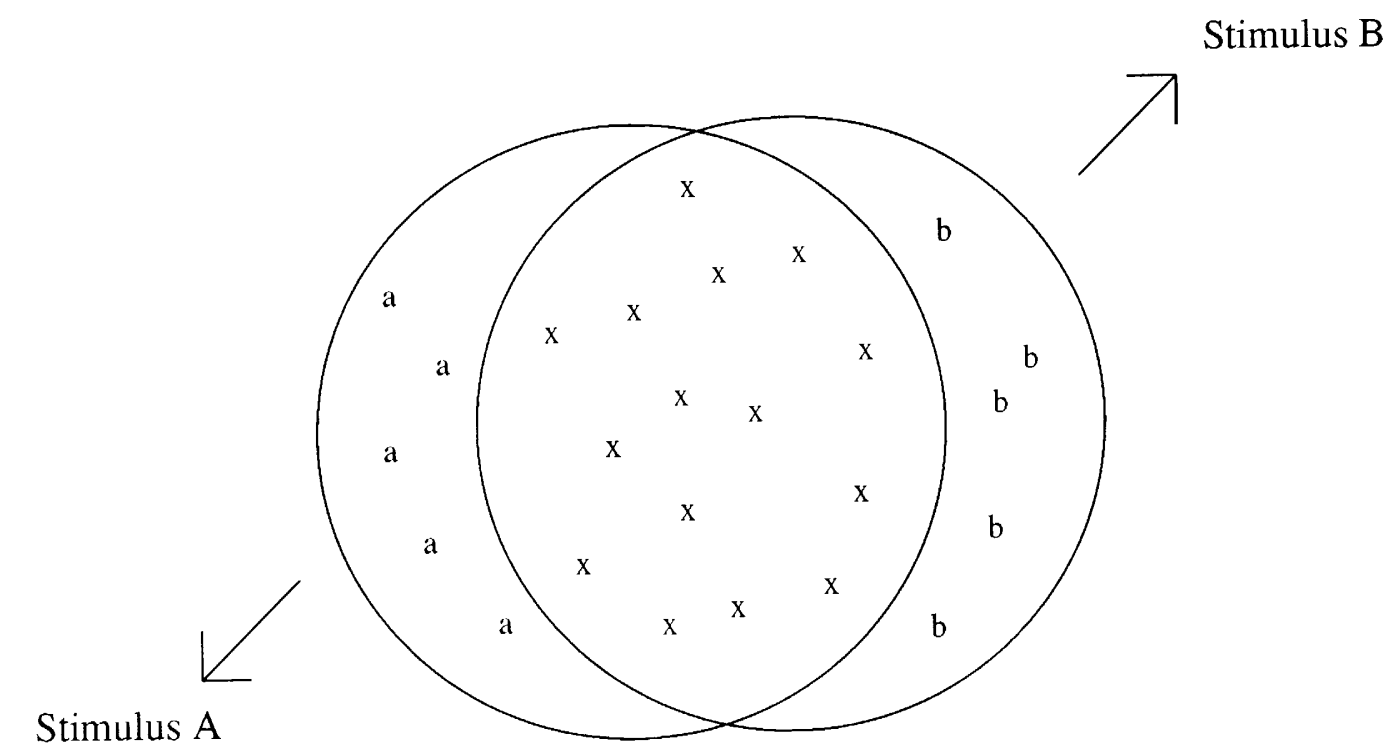

Figure 1. A representation of two stimuli, A and B, which are comprised of many elements, some of which are unique to each stimulus ( $\mathrm{a}$ and $\mathrm{b}$ ) and some of which are common to both stimuli (x).

candidate processes that may be involved to reduce the salience of features held in common between two similar stimuli during preexposure.)

Perceptual learning is therefore a phenomenon of reduced generalisation in which the subject responds less to the common features and more to the unique features of two highly similar and familiar stimuli following preexposure. Our prediction was that normal adults would benefit from preexposure in the subsequent discrimination phase by discriminating between preexposed stimuli better than between non-preexposed stimuli, replicating previous studies. By contrast, individuals with autism would show even better discrimination between two previously exposed stimuli compared to the control group because our hypothesis was that individuals with autism process unique features better and common features worse than other individuals. Furthermore, and for the same reason, it was predicted that people with autism would show superior discrimination between nonpreexposed stimuli compared to control subjects.

\section{Method}

\section{Participants}

Two groups of adults participated: a group of 8 highfunctioning adults with autism and a control group of 10 nonautistic adults. The participants with autism constitute an unusually able sample. Some were employed and all were living independently. They had all been diagnosed by clinicians according to established criteria for autism, such as those specified in DSM-IV (American Psychiatric Association, 1994). Adults in the control group were recruited by advertisement within the office building of a computer software company in the Cambridge area. All participants were assessed for verbal and spatial ability using the vocabulary and block design subtests of the Wechsler Adult Intelligence Scale (WAIS-R; Wechsler, 1981). The mean scaled scores and standard deviations obtained by each group on each subtest and mean chronological ages of each group are presented in Table 1. Independent $t$-tests conducted on scaled scores showed that the two groups did not differ in their performance either on the vocabulary subtest $[t(16)=0.553, p=.59]$ or the block design subtest $[t(16)=0.09, p=.93]$.

\section{Apparatus}

The stimuli were generated by an Acorn Risc PC computer and displayed on a 14-inch colour monitor. Subjects sat 2 feet from the monitor, and responded to stimuli by pressing one of two keys on a standard keyboard (either the "." key with the right hand or the " $z$ " key with the left hand). In order to avoid accidental presses of other irrelevant keys, the keyboard was covered with a hard black plastic cover with two openings allowing access to the two response keys.

\section{Stimuli}

Each stimulus consisted of seven circles superimposed on a rectangular background. Each circle was coloured with a "beachball" pattern in red and yellow, and measured $1.5 \mathrm{~cm}$ in diameter (95 pixels). The rectangular background was coloured blue and measured $22 \mathrm{~cm}$ wide and $16 \mathrm{~cm}$ high (1400 by 1000 pixels). There were 8 such stimuli in total, 4 of which were

Table 1

Subject Characteristics

\begin{tabular}{lrccc}
\hline Group & $N$ & Chronological age & Vocabulary subtest $^{\mathrm{a}}$ & Block design subtest $^{\mathrm{a}}$ \\
\hline Autistic & 8 & 28 yrs $9 \mathrm{mths}$ & $10.75(3.77)$ & $12.63(4.81)$ \\
Control & 10 & 28 yrs 6 mths & $11.6(2.76)$ & $12.8(3.88)$ \\
\hline
\end{tabular}

${ }^{a}$ Scaled scores: values enclosed in parentheses represent standard deviations. 
Table 2

The Design of the Experiment, Showing the Different Types of Discrimination Problem Presented to the Subjects During Each Phase

\begin{tabular}{ll}
\hline Preexposure phase & Test phase \\
\hline A1 versus A2 & A3 versus A4 \\
or & followed by \\
B1 versus B2 & B3 versus B4 \\
& or \\
& B3 versus B4 \\
& followed by \\
& A3 versus A4 \\
\hline
\end{tabular}

classified as group A stimuli and 4 as group B stimuli. Group A and group B stimuli differed with respect to the general pattern created by the positions of the 7 circles on the screen. These 8 stimuli are shown in Fig. 2.

Each of the circles in stimuli A1 and B1 has been numbered, although these numbers did not appear on the stimuli presented to subjects. Stimuli A1 and A2 were the same with respect to the position on the screen of circles 1,5 , and 7 and differed with respect to the positions of the remaining four circles. Two further group A stimuli, A3 and A4, were the same as A1 and A2 with respect to the positions of circles 1,5 , and 7 but were different from each other and from stimuli A1 and A2 with respect to the positions of the remaining circles. Similarly, the four group B stimuli were the same with respect to the positions of three circles (circles 3, 4, and 6 in Fig. 2: bottom panel) but each differed with respect to the positions of the remaining four circles.

\section{Design}

During the preexposure phase, participants were required to learn to discriminate between either stimuli $\mathrm{A} 1$ and $\mathrm{A} 2$ or stimuli B1 and B2. During the test phase, all subjects were given two further discrimination problems, one between stimuli A3 and A4 and the other between B3 and B4. Hence, for half the participants in each group, the familiar stimuli were A3 and A4 and the novel stimuli were B3 and B4. For the remaining subjects, the familiar stimuli were B3 and B4 and the novel stimuli were A3 and A4. In any perceptual learning experiment, it is important to counterbalance stimuli in this way, since if only one type were preexposed, any enhancement of discrimination of this type compared to the novel type could result merely from the familiar stimuli being less similar than the novel stimuli. The counterbalancing therefore ensured that an enhancement in discrimination learning about familiar stimuli resulted from a reduction in the salience of common features rather than any other reason.

Order of discrimination problem (familiar stimuli first or novel stimuli first) during the test phase was also counterbalanced between participants. The reason for this was that performance on the second discrimination problem could either be enhanced as a result of generalised practice at the task during the first problem, or could be reduced as a result of fatigue. Counterbalancing therefore removed these potential sources of confound. This design is outlined in Table 2 .

\section{Procedure}

There were nine separate stages of the experimental session, details of which are given below. Each stage was separated by the appearance of a blank screen with the message "Click mouse to continue" printed at the top of the screen. These "rest" periods allowed the experimenter to deliver instructions concerning the next phase of the experiment and for the participant to rest if required. At the start of the session, the participants were instructed that throughout the task they would be shown two different pictures, presented separately, and that their aim was to discover which picture went with which button.

With the exception of the stimuli presented in Stage 2, all stimuli were presented for a maximum of 4 seconds. In addition, with the exception of those presented in the test phase (Stages 8 and 9), all stimuli were presented for a minimum of 2 seconds. The reason for this was to encourage participants to study the stimulus rather than respond immediately following stimulus onset. The start of each trial was signalled with the presentation of a fixation stimulus. This was a white cross $(1 \mathrm{~cm} \times 1 \mathrm{~cm})$ displayed centrally on a uniform dark grey background for $0.5 \mathrm{sec}$. The stimuli were presented immediately after the offset of this fixation stimulus. Trials were terminated after 4 seconds had elapsed or when the participant pressed a response key, whichever was the sooner. In all stages other than 8 and 9, if participants made a response within 2 seconds of trial onset, the trial was terminated immediately after 2 seconds had elapsed. Upon trial termination, the screen returned to uniform dark grey and a feedback message ("correct", "incorrect", "you did not respond in time", or "you have pressed the wrong key") was immediately displayed in white letters in the centre of the screen. The message was displayed for $1.5 \mathrm{sec}$, after which the screen returned to a dark grey for $0.5 \mathrm{sec}$ before the small cross appeared to signal the next trial. Error data were recorded on each trial in the preexposure phase and both reaction time and error data were recorded on each trial during the test phase.

Stage 1. In order to familiarise the participants with the task, the session began with simple discrimination training in which participants learned to press the left key in response to a blue triangle and the right key in response to a red circle. The stimuli were presented successively, drawn in the middle of the screen, and measured $3 \mathrm{~cm}$ high and $3 \mathrm{~cm}$ wide $(180 \times 180$ pixels). There were 10 trials in total, 5 of the red circle and 5 of the blue triangle, and trial types were randomly intermixed.

Stage 2. This was an instruction stage designed to familiarise the participants with the nature of the "beachball" stimuli. Two such "dummy" stimuli were drawn side by side on the screen. Each stimulus was a rectangle superimposed with seven "beachball" circles. The stimuli differed with respect to the position of four circles. These differences were pointed out to participants and it was explained that in subsequent phases of the session only one stimulus would appear on the screen at any one time. Participants were told that they should therefore try to remember where the circles were on each stimulus in order to tell the two stimuli apart.

Stage 3. This was a discrimination training phase involving the two "dummy" stimuli which had been presented in stage 2 . Participants were instructed to find which stimulus went with which key. The two stimuli were presented successively. There were 42 trials in total, with equal numbers of each stimulus. The order in which the two stimuli appeared was predetermined. During the first 10 trials, each stimulus appeared on alternate trials. The purpose of this ordering was to provide the opportunity for participants to compare the stimuli in order to assist detection of their differences. During the next 12 trials, 2 successive trials were of 1 stimulus followed by 2 successive trials of the other stimulus. For the remaining 20 trials, the 2 stimuli were randomly intermixed.

Stage 4. This was another demonstration stage in which participants were shown those stimuli that would appear during preexposure (either A1 and A2 or B1 and B2). There were four trials in total, two showing one stimulus and two showing the other. The participants were not required to respond and, whilst 

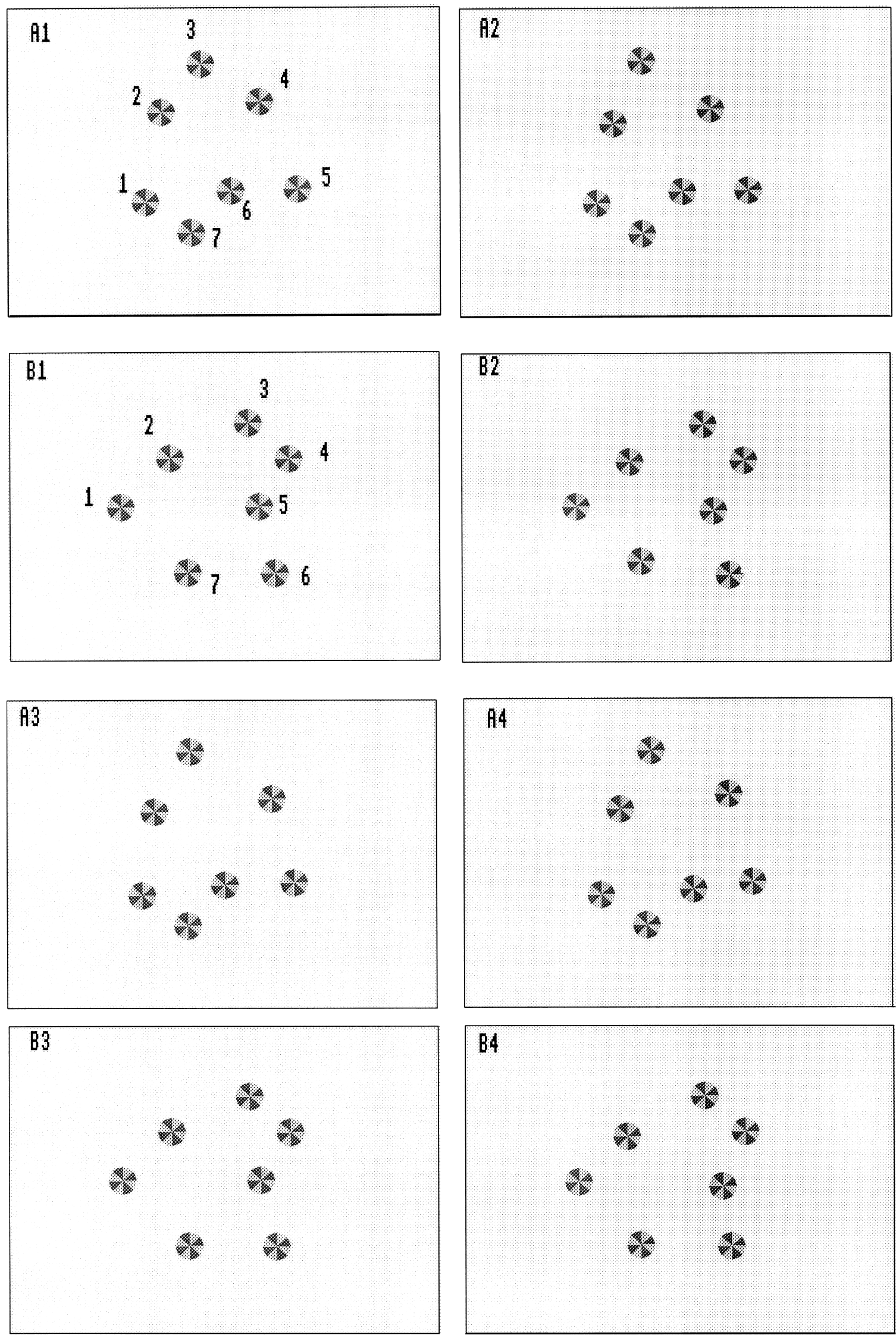

Figure 2. Stimuli used during the preexposure phase are shown in the top half of the figure and the test stimuli are shown in the bottom half. The circles in stimuli A1 and B1 have been numbered but these numbers did not appear on the stimuli shown to the subjects. Circles 1, 5, and 7 in stimulus A1 appeared in the same positions on the screen in stimuli A2, A3, and A4. Circles 3, 4, and 6 in stimulus B1 appeared in the same positions in stimuli B2, B3, and B4. The positions of the remaining circles were different for each stimulus. 
being shown the stimuli, were told that they differed with respect to the position of the circles and that their aim was to find which stimulus went with which key.

Stage 5. Participants were given 20 practice trials, 10 trials showing 1 preexposure stimulus and 10 showing the other. Trial types were randomly intermixed. These trials acted, of course, as preexposure trials, and differed from Stage 6 only in that, in this stage, the experimenter gave verbal reminders that the task was to discover which stimulus went with which key.

Stage 6 (preexposure phase). During this phase, half the participants learnt a discrimination between $\mathrm{A} 1$ and $\mathrm{A} 2$ and the remaining participants learnt a discrimination between $\mathrm{B} 1$ and B2 (see Table 2, preexposure phase). There were 80 trials in total, which were split into 2 blocks of 40 trials. Participants could therefore take a break between blocks, if required. There were equal numbers of each stimulus in each block and trial types were randomly intermixed.

Stage 7. The purpose of this stage was to separate the preexposure phase from the text phase. Participants were given the simple discrimination problem that had occurred in Stage 1, using the same stimuli and involving the same number of trials.

Stage 8 and 9 (test phase). In the two test blocks, half the participants were given a discrimination problem between stimuli that were similar to those preexposed (preexposed condition), followed by a discrimination problem between two wholly novel stimuli (non-preexposed condition). For the remaining participants, this order was reversed (see Table 2, test phase). There were 40 trials in each block and trial types within each block were randomly intermixed. Immediately prior to the preexposed condition, participants were told that the stimuli that would appear would look similar to those seen previously in the session. They were also warned that the way in which the previous pictures had differed may or may not be the way in which the new pictures differ. They were advised to therefore look at all the circles in order to try to solve the discrimination problem.

\section{Results}

The ability of each group to master the discrimination problem set in the preexposure phase was analysed prior to the results of the test phase. The results of the preexposure phase are presented in the graph in Fig. 3, which shows accuracy scores for each group of participants. The data from the 20 practice trials and 80 subsequent training trials have been separated into 10 trial blocks, each trial block representing the average of 10 trials. The graph indicates that the discriminations were learned at roughly the same rate by each group. This was supported by an ANOVA, with a between-subjects factor of group and a within-subjects factor of block, which revealed no difference between group, $F<1$, a significant effect of trial block, $F(9,144)=5.291$, $p<.0001$, and no interaction, $F<1$.

However, the graph indicates some difference between groups by the last trial block, where the group with autism appeared to have reached a higher level of learning. This was confirmed by ANOVA, which compared the performance of the groups on the first and last trial blocks only. Although there was no overall effect of group, $F<1$, there was a significant effect of trial block, $F(1,16)=31.66, p<0.0001$, and a significant group by trial block interaction, $F(1,16)=4.67, p<.05$. Simple effects analysis of this interaction showed that there was a significant difference between groups on the last trial block, $F(1,16)=4.7, p<.04$, with the average score of the group with autism being higher than that of the control group ( 0.95 and 0.79 respectively).

Figure 4 presents graphs of the results of the test phase. The left panel presents the accuracy scores from the preexposure condition and the right panel shows the scores from the non-preexposed condition. In each graph, the data from the 40 trials of each condition are presented separately, in 4 trial blocks, each block representing the average of 10 trials. The data from the control participants indicates a perceptual learning effect in that the stimuli in the preexposed condition were discriminated more accurately than those in the non-preexposed condition. By contrast, the performance of the participants with autism was roughly equivalent in the two conditions.

The average accuracy score across 40 trials was calculated for each participant in each condition and these data were analysed by a mixed ANOVA with three between-subjects factors and one within-subjects factor. The first between-subjects factor was group. The second was type of preexposed and non-preexposed stimuli (i.e. group A preexposed and group B non-preexposed or vice versa). This factor was included in the analysis to assess whether group A stimuli and group B stimuli were equated for discriminability per se. The third was order of test discrimination problem (preexposed condition first or second). The within-subjects factor was type of test discrimination problem (preexposed or non-preexposed).

The main effects of group, type of preexposed or nonpreexposed stimuli, and order were not significant, $F$ s $<1$. The lack of effect of type of stimuli and order indicates that any difference in performance between preexposed and non-preexposed conditions was not due to stimulus specific factors or practice per se. There was a main effect of type of test discrimination problem, $F(1,10)=7.647, p<.02$, where stimuli in the preexposed condition were better discriminated than stimuli in the non-preexposed condition (average accuracy scores were 0.80 and 0.69 respectively) and a significant interaction between group and type of test discrimination problem, $F(1,10)=15.16, p<.003$.

Simple effects analysis of this interaction revealed that control participants performed more accurately in the preexposed condition than in the non-preexposed condition, $F(1,10)=25.4, p<.001$. No such perceptual learning effect was observed in the group with autism since there was no difference in their performance between the preexposed and non-preexposed conditions, $F<1$. Hence, our prediction that participants with autism would show an enhanced perceptual learning effect was not borne out. Further simple effects analysis revealed why. First, the participants with autism discriminated between stimuli in the non-preexposed condition significantly better than the control participants, $F(1,18)=$ 4.56, $p<.05$. Second, the participants with autism discriminated between stimuli in the preexposed condition significantly worse than control participants, $F(1,18)=5.29, p<.04$.

The fact that participants with autism did not show a perceptual learning effect suggests that both the stimuli in the non-preexposed and preexposed conditions of the test phase were regarded as novel. If this is true, the performance of participants with autism on the first 40 trials of the novel discrimination problem given in the 


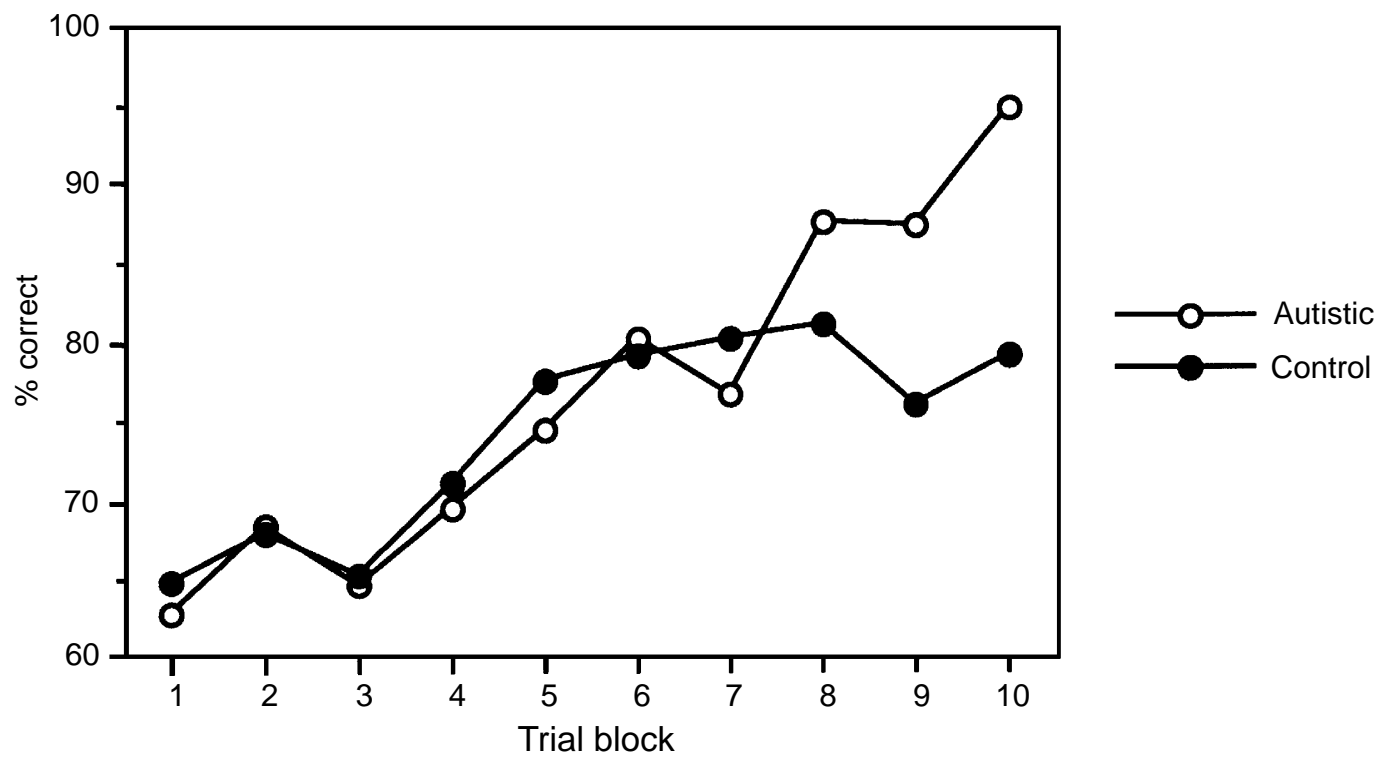

Figure 3. Average accuracy scores for each group on the discrimination problem during the preexposure phase, showing superior performance by adults with autism in the final three trial blocks.

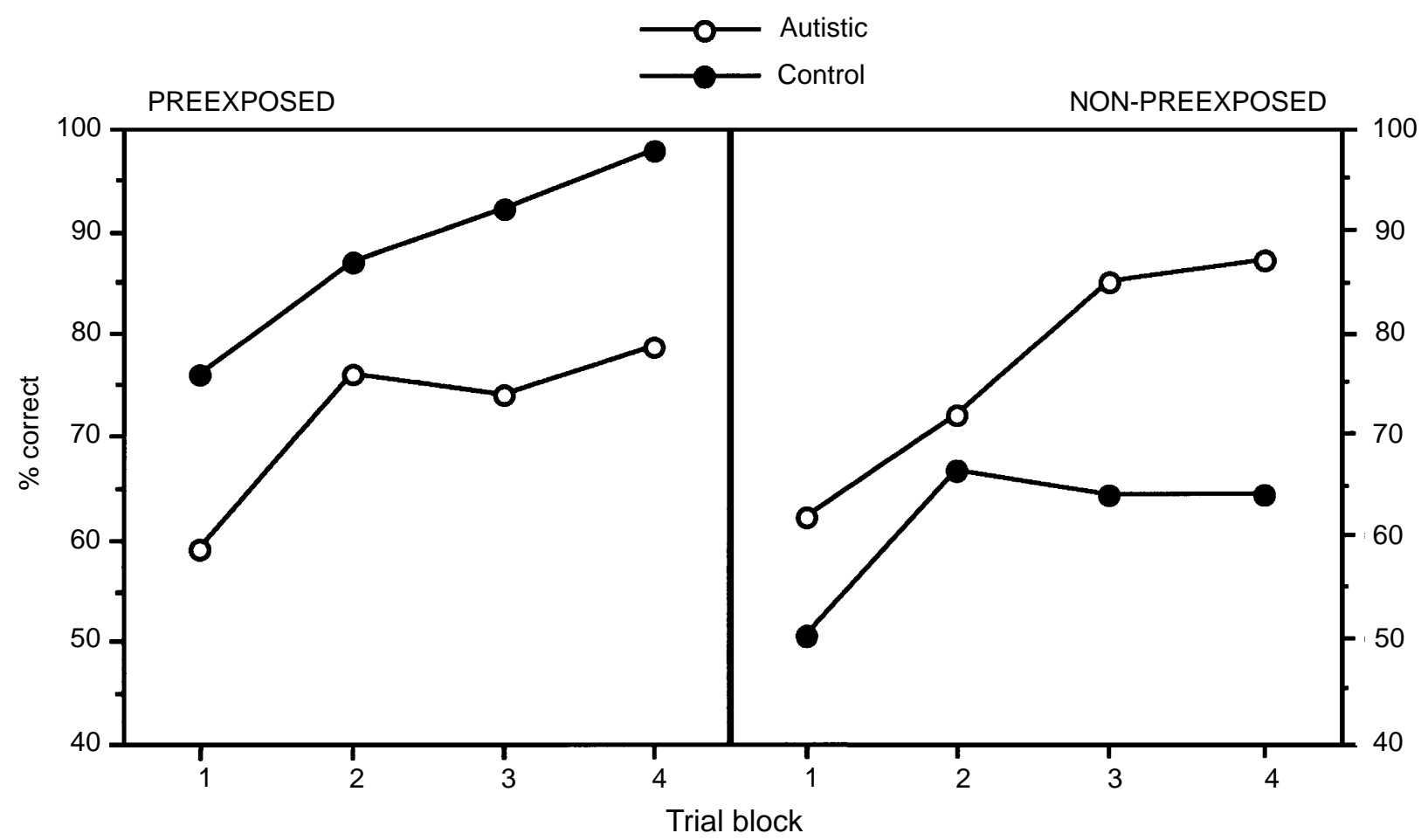

Figure 4. Average accuracy scores for each group during the test phase. Adults with autism discriminated stimuli in the nonpreexposed condition better than nonautistic adults and nonautistic adults discriminated stimuli in the preexposed condition better than adults with autism.

preexposure phase should be comparable to their performance on the two conditions of the test phase, since all three discrimination problems would effectively be novel problems. By contrast, if control participants regarded the stimuli in the preexposed condition of the test phase as familiar (as suggested by the perceptual learning effect in their performance) their performance on the first 40 trials of the novel problem in the preexposure phase should be comparable only to their performance on the novel problem in the non-preexposed condition of the test phase. As a test of this, a further ANOVA was conducted comparing the performance of each group in the first 40 trials of the preexposure phase with performance in the 40 trials of the preexposed and nonpreexposed conditions of the test phase.

The analysis contained one between-subjects factor of group and one within-subjects factor of discrimination problem (preexposure phase, preexposed and non- 


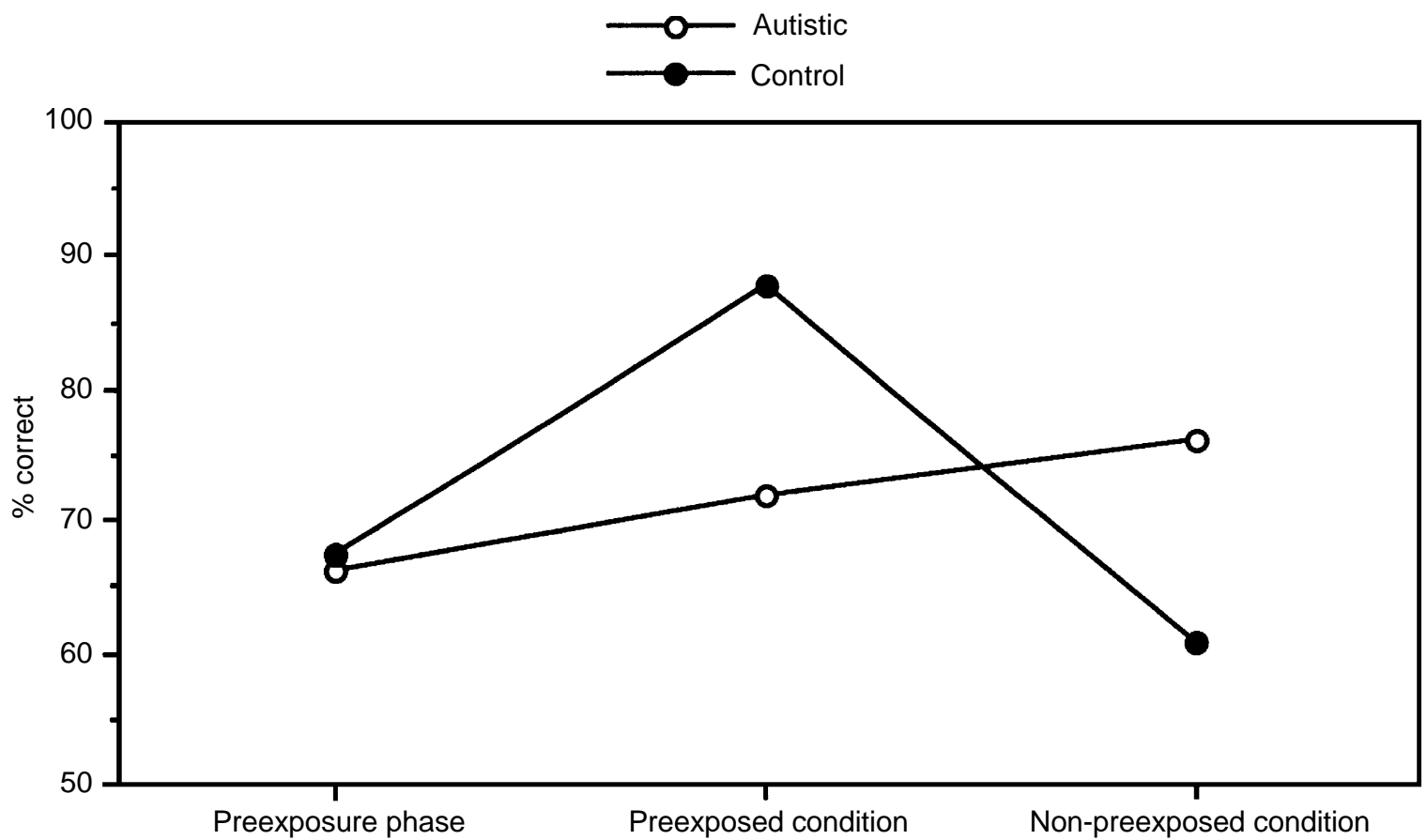

Figure 5. Average accuracy scores for each group in the first 40 trials of the preexposure phase, the preexposed condition, and the non-preexposed conditions of the test phase. Participants with autism performed with the same accuracy in all three conditions whereas control adults were more accurate in the preexposed condition of the test phase than in the other two conditions.

preexposed). There was no effect of group, $F<1$, and a main effect of discrimination problem, $F(2,32)=4.28$, $p<.03$. Newman-Keuls pairwise comparisons revealed that performance in the preexposed condition was significantly better $(p<.05)$ compared to the other two conditions, which did not differ significantly from one another. Furthermore, there was a significant interaction between group and discrimination problem, $F(2,32)=5.4, p<.01$.

This interaction is illustrated in Fig. 5. Simple effects analysis revealed that there were no differences in performance between the two groups in the first 40 trials of the preexposure phase $(F<1)$, that the performance of the control participants was more accurate than that of the participants with autism in the preexposed condition of the test phase, $F(1,42)=3.82, p<.05$, and that the performance of the participants with autism was more accurate than the control participants in the nonpreexposed condition, $F(1,42)=4.01, p<.05$. More importantly, there was an effect of discrimination problem in the data from the control participants, $F(2,32)=$ $9.59, p<.01$, but no such effect in the data from the participants with autism, $F(2,32)=1.04$. NewmanKeuls pairwise comparisons revealed that the effect in the data from control participants arose because their performance was significantly more accurate in the preexposed condition than in the other two conditions $(p<.05)$, and that performance in the preexposure phase did not differ statistically from performance in the nonpreexposed condition in the test phase. Newman-Keuls pairwise comparisons of the data from the participants with autism revealed no significant differences between the three conditions. This supports the impression that all three problems, including the problem in the preexposed condition, were regarded as novel by the participants with autism.

Reaction time data taken during the test phase were analysed by ANOVA with three between-subjects factors of group, type of preexposed and non-preexposed stimuli, and order of test discrimination problem, and one withinsubjects factor of type of test discrimination problem. The only significant effect, $F(1,10)=12.24, p<.006$ was that the mean reaction time of the control group was significantly faster than the group with autism (134 and 184 centiseconds, respectively). There were no other main effects and no interactions.

\section{Discussion}

Adults with autism performed better than the control group by the end of the preexposure phase and significantly more accurately at test in the non-preexposed condition than nonautistic adults. These results suggest that individuals with autism are better able than nonautistic individuals to solve novel discrimination problems involving highly similar stimuli. This is consistent with the hypothesis that people with autism process unique features better and process common features worse relative to nonautistic people. Thus, the features shared in common between the stimuli involved in these discrimination problems did not produce the same degree of generalisation in the group with autism as in the group of nonautistic individuals. This may underlie the poor generalisation of learning frequently observed in autism.

But what is the reason for the relatively poor performance by the group with autism in the preexposed 
condition compared to the control group? There are at least three theories that present possible reasons why the enhancement of discrimination learning in the preexposed condition was not observed in the group with autism-weak central coherence, reduced attentionswitching, and our hypothesis of differential processing of unique and common features in autism.

The idea of weak central coherence is that individuals with autism show a reduced ability to draw together the features of a stimulus into a coherent whole (Frith, 1989; Frith \& Happé, 1994) and, as a result, do not readily perceive the overall gestalt of a stimulus or figure. What ramifications does this possibility have given the types of stimuli used here? It may have been the case that the control participants may have processed each stimulus at the global level, by which is meant they perceived each stimulus as a gestalt or pattern created by all seven circles, thereby solving the discriminations on the basis of this gestalt. This overall pattern would, of course, have been different in all of the stimuli. If the group with autism were unable to perceive the pattern created by all seven circles, their ability to solve the discriminations would have to rely on detecting differences at a more local area of the stimulus, perhaps involving the relative spatial positions of just two or three circles. However, there is no obvious reason why this by itself would confer a disadvantage relative to perceiving global differences unless it was argued that there was greater similarity between the relative positions of a few circles in a local area than between the relative positions of all seven circles. But this would not explain the superior ability of the group with autism to discriminate between stimuli in the preexposure phase and between novel stimuli in the test phase.

Furthermore, there is at least another reason for doubting this possibility as a plausible explanation for the difference between subject groups. Perception of a global pattern in each stimulus by the control group would predict not a strong but a weak, or reduced, perceptual learning effect-discrimination learning about familiar stimuli would not be enhanced following preexposure but be equivalent to learning about the novel stimuli. Perceptual learning effects occur only when the preexposed stimuli and the stimuli to be discriminated between in a subsequent test phase hold features in common. While the positions of three circles were held in common between the two stimuli presented during the preexposure phase and the two stimuli presented during the test phase, the global pattern of all seven circles would have been unique to each of the four stimuli. There would therefore be very little (if any) beneficial effect of preexposure on subsequent discrimination learning in the test phase. Given that a strong perceptual learning effect was observed in the control group, we can conclude that their performance was not based on the perception of an overall pattern in each stimulus.

The second possible reason for the relatively poor performance by the group with autism in the preexposed condition compared to the control group appeals to the idea of reduced attention switching and attentional flexibility. For example, Courchesne and his colleagues (Courchesne, Townsend, Ashoomoff, YeungCourchesne, et al., 1994; Courchesne, Townsend, Ashoomoff, Saitoh, et al., 1994) have found that in- dividuals with autism are less able to shift attention between different stimuli. Similarly, individuals with autism show impairments on some tests of executive function that tap attentional flexibility (Hughes, Russell, \& Robbins, 1994; Ozonoff, in press; Russell, 1997). Thus, it may have been the case that during preexposure, both groups had focused on just one area of the screen in order to detect differences in relative spatial positions of two or three circles. At test, however, there may have been very little difference in relative spatial position between the two stimuli in this particular area of the screen. In this case, participants would have had to shift the focus of their attention to other areas of the stimuli in order to solve the discrimination problem. If the participants with autism were less able than the control group to shift attention to the relevant area of stimuli, this could account for the lack of enhanced discrimination learning in the group with autism in the preexposed condition.

Our final suggestion is consistent with the hypothesis that individuals with autism process unique features well and common features poorly compared to nonautistic individuals. Perceptual learning is dependent upon the familiarity of the test stimuli as a result of preexposure and thus on some form of "recognition" occurring at the test phase. Hence, an enhancement of discrimination learning at the test phase requires, in part, some generalisation of learning from the preexposure phase to the test phase. Indeed, the better performance of the control group on the familiar stimuli in the test phase compared to performance in the preexposure phase is evidence of such generalisation. Recall that three of the circles in the text stimuli were in the same relative positions as in the preexposure stimuli; recognition of this would mean that the nonautistic participants needed to learn only about the positions of the remaining four circles, allowing their learning to proceed more quickly and to reach a higher level in the available 40 test trials than if learning about wholly novel stimuli.

In contrast, the participants with autism learnt about the familiar stimuli at about the same rate and to about the same level as the preexposure stimuli in the equivalent first 40 trials of the preexposure phase. Thus, there was no evidence of generalisation of learning about the relative positions of the three circles from the preexposure phase to the test phase by the adults with autism. This would suggest that these familiar stimuli in the test phase were regarded as wholly novel stimuli.

Thus, generalisation depends on the extent to which a subject processes or recognises common features. Any reduction in the ability to process such common features during preexposure could reduce the enhancement in discrimination between familiar stimuli during the test phase normally observed following preexposure-it could reduce a perceptual learning effect in the way that we observed in the performance of the adults with autism. In short, although poor processing of features held in common between stimuli will assist learning novel discrimination problems involving highly similar stimuli, this could also prevent an enhancement of discrimination between familiar stimuli during a subsequent test phase.

It would therefore appear that two hypotheses, one of reduced generalisation and the other of reduced attention switching or poor attentional flexibility in autism, can 
account for the lack of enhancement of discrimination learning about preexposed stimuli in the text phase. The advantage of the reduced generalisation hypothesis is that it can additionally account for the better performance by the individuals with autism by the end of the preexposure phase and in the non-preexposed condition during the test phase, whereas the attention switching hypothesis cannot.

But how might these two accounts be dissociated empirically? It would be possible to design a task where people must attend to the same area of the screen in both the preexposure and test phases. The attention switching hypothesis would not predict a difference between groups, because there would be no requirement to attend to a different screen location at the test phase. By contrast, the reduced generalisation hypothesis would still predict that learning about stimuli in the preexposure phase would not transfer as well in individuals with autism compared to nonautistic individuals so that the perceptual learning effect would be reduced. This hypothesis would also predict, of course, that individuals with autism would learn discriminations involving novel stimuli, as in the non-preexposed condition, better than other individuals.

In summary, the idea of relatively good processing of unique features and relatively poor processing of common features by autistic compared to nonautistic individuals is supported by the pattern of discrimination performance we observed during a perceptual learning task. It may, therefore, be this process that underlies reduced generalisation of learning, which is specific to autism. Clearly, other tests are required to evaluate this hypothesis further. Suitable tests would, of course, be any which require differential processing of unique and common features. It is of interest that one can demonstrate that, under certain conditions, abnormalities in autism produce superior performance - in this case, enhanced discrimination of novel, highly similar stimuli. Although autism is usually characterised as a disability, it is also clear that experiments such as the present one reveal that autism can also be characterised as an unusual pattern of strengths. Findings of specific superiority such as these rule out trivial explanations in terms of distractibility, comprehension, etc., and instead suggest the existence of cognitive differences in autism.

Acknowledgements - Kate Plaisted was supported by the Pinsent-Darwin Fund, University of Cambridge, and a Research Fellowship from St John's College, Cambridge, and Michelle O'Riordan and Simon Baron-Cohen were supported by the MRC during the period of this work. Grateful thanks to Simon Killcross and Mike Aitken for important background discussion and comments, to Therese Jolliffe for her generous help in recruiting the subjects for this study, to Jim Russell for comments on an earlier draft of this manuscript, and to the individuals and their families who took part.

\section{References}

Aitken, M. R. F., Bennett, C. H., McLaren, I. P. L., \& Mackintosh, N. J. (1996). Perceptual differentiation during categorisation learning in pigeons. Journal of Experimental Psychology: Animal Behaviour Processes, 22, 43-50.
American Psychiatric Association. (1994). Diagnostic and statistical manual of mental disorders (4th Edn.). Washington, DC: Author.

Attneave, F. (1957). Transfer of experience with a class-schema to identification-learning of patterns and shapes. Journal of Experimental Psychology, 54, 81-88.

Baron-Cohen, S., Leslie, A. M., \& Frith, U. (1985). Does the autistic child have a "theory of mind"? Cognition, 21, 37-46.

Courchesne, E., Townsend, J., Ashoomoff, N. A., YeungCourchesne, R., Press, G., Murakami, J., Lincoln, A., James, H., Saitoh, O., Haas, R., \& Schreibman, L. (1994). A new finding in autism: Impairment in shifting attention. In S. H. Broman \& J. Grafman (Eds.), Atypical cognitive deficits in developmental disorders: Implications for brain function (pp. 101-137). Hillsdale, NJ : Lawrence Erlbaum.

Courchesne, E., Townsend, J., Ashoomoff, N. A., Saitoh, O., Yeung-Courchesne, R., Lincoln, A., James, H., Haas, R., Schreibman, L., \& Lau, L. (1994). Impairment in shifting attention in autistic and cerebellar patients. Behavioral Neuroscience, 108, 848-865.

Donnellan, A. M., \& Mirenda, P. L. (1983). A model for analysing instructional components to facilitate generalisation in severely handicapped students. Journal of Special Education, 17, 317-331.

Frith, U. (1989). Autism: Explaining the enigma. Oxford: Blackwell.

Frith, U., \& Happé, F. G. E. (1994). Autism: Beyond "theory of mind". Cognition, 50, 115-132.

Gibson, J. J., \& Gibson, E. J. (1955). Perceptual learningdifferentiation or enrichment? Psychological Review, 62, $32-41$.

Gibson, E. J. (1969). Principles of perceptual learning and development. New York: Appleton-Century-Crofts.

Gibson, E. J., \& Walk, R. D. (1956). The effect of prolonged exposure to visually presented patterns on learning to discriminate them. Journal of Comparative and Physiological Psychology, 49, 239-242.

Hall, G. (1991). Perceptual and associative learning. Oxford: Clarendon Press.

Hughes, C., Russell, J., \& Robbins, T. W. (1994). Evidence for executive dysfunction in autism. Neuropsychologia, 32, 477492.

Lovaas, O. I., Koegel, R. L., \& Schreibman, L. (1979). Stimulus overselectivity in autism: A review of research. Psychological Bulletin, 86, 1236-1254.

Lovaas, O. I., Schreibman, L., Koegel, R. L., \& Rhem, R. (1971). Selective responding by autistic children to multiple sensory input. Journal of Abnormal Psychology, 77, 211-222.

Lubow, R. E. (1973). Latent inhibition. Psychological Bulletin, 79, 398-407.

Mackintosh, N. J., Kaye, H., \& Bennett, C. H. (1991). Perceptual learning in flavour aversion conditioning. Quarterly Journal of Experimental Psychology, 43B, 297-322.

McLaren, I. P. L., Kaye, H., \& Mackintosh, N. J. (1989). An associative theory of the representation of stimuli: Applications to perceptual learning and latent inhibition. In R. G. M. Morris (Ed.), Parallel distributed processing: Implications for psychology and neurobiology (pp. 102-130). Oxford: Clarendon Press.

McLaren, I. P. L., Leavers, H. J., \& Mackintosh, N. J. (1994). Recognition, categorisation and perceptual learning. In C. Umiltà \& M. Moscovitch (Eds.), Attention and performance $X V$ : Conscious and nonconscious information processing (pp. 889-909). Cambridge, MA: MIT Press.

Mirenda, P. L., \& Donnellan, A. M. (1987). Issues in curriculum development. In D. J. Cohen, A. M. Donnellan, \& R. Paul (Eds.), Handbook of autism and pervasive developmental disorders. New York: John Wiley \& Sons. 
Ozonoff, S. (in press). Components of executive dysfunction in autism and other disorders. In J. Russell (Ed.), Autism as an executive disorder. Oxford: Oxford University Press.

Ozonoff, S., \& Miller, J. N. (1995). Teaching theory of mind-a new approach in social skills training for individuals with autism. Journal of Autism and Developmental Disorders, 25, 415-433.

Pearce, J. M., \& Redhead, E. S. (1993). The influence of an irrelevant stimulus on two discriminations. Journal of Experimental Psychology: Animal Behavior Processes, 19, 180-190.

Perner, J., Frith, U., Leslie, A. M., \& Leekam, S. R. (1989). Exploration of the autistic child's theory of mind: Knowledge, belief and communication. Child Development, 60, 689-700.

Rincover, A., \& Koegel, R. L. (1975). Setting generality and stimulus control in autistic children. Journal of Applied Behaviour Analysis, 8, 235-246.
Russell, J. (1997). How executive disorders can bring about an inadequate "theory of mind". In J. Russell (Ed.), Autism as an executive disorder (pp. 256-304). Oxford: Oxford University Press.

Swettenham, J. G. (1996). Can children with autism be taught to understand false belief using computers? Journal of Child Psychology and Psychiatry, 37, 157-165.

Wechsler, D. (1981). Wechsler Adult Intelligence ScaleRevised. Cleveland, $\mathrm{OH}$ : The Psychological Corporation.

Wilhelm, H., \& Lovaas, O. I. (1976). Stimulus overselectivity: A common feature in autism and mental retardation. American Journal of Mental Deficiency, 81, 227-241.

Woods, T. S. (1987). The technology of instruction: A behavior analytic approach. In D. J. Cohen, A. M. Donnellan, \& R. Paul (Eds.), Handbook of autism and pervasive developmental disorders. New York: John Wiley \& Sons.

Manuscript accepted 12 September 1997 
Copyright $\odot 2003$ EBSCO Publishing 